\title{
ARTROPLASTIA TOTAL DE DISCO CERVICAL COM PRÓTESE DE BRYAN. RESULTADOS CLÍNICOS E FUNCIONAIS
}

\author{
CERVICAL TOTAL DISC ARTHROPLASTY WITH BRYAN DISC. \\ CLINICAL AND FUNCTIONAL OUTCOMES
}

\author{
ARTROPLASTÍA CERVICAL TOTAL CON PRÓTESIS DE BRYAN. \\ RESULTADOS CLINNICOS Y FUNCIONALES
}

Eduardo Machado de Menezes ${ }^{1}$, Rafael Ozório Rocha', Aline Aparecida Depiante Moreira', Deusdeth Gomes Nascimento², antonio Eulálio Pedrosa Araujo ${ }^{3}$, luiz Carlos Santeli Mala ${ }^{3}$

\begin{abstract}
RESUMO
Objetivo: Avaliar resultados da artroplastia total disco cervical com a prótese de Bryan para tratamento da cervicobraquialgia (radiculopatia) e da mielopatia compressiva. Métodos: No período de 2002 a 2007, a equipe da CECOL operou 65 pacientes. Somente 28 pacientes foram localizados em 2010 para uma nova coleta de dados. Foi feita avaliação pré e pós-operatória, utilizando-se o questionário CSOQ (Cervical Spine Outcomes Questionnaire). Os critérios de Odom foram utilizados somente na avaliação pós-operatória. Ambos foram traduzidos e adaptados para cultura local. Resultados: Houve melhora funcional e sintomática significativa na maioria dos pacientes. A atenuação da dor cervical (axial) e braquial (radicular) foi semelhante. Os critérios Odom indicaram 82,1\% de bons e ótimos resultados, $10 \%$ satisfatórios e $7 \%$ ruins. Houve apenas uma complicação (3\%), que foi revertida com a artrodese anterior. Conclusões: A artroplastia total de disco cervical demonstrou ser um método seguro e eficaz para tratamento de casos selecionados de hérnia de disco cervical associada a radiculopatia e/ou mielopatia a curto e médio prazo.
\end{abstract}

Descritores: Artroplastia; Artrodese; Compressão da medula espinal; Degeneração do disco intervertebral; Radiculopatia.

\begin{abstract}
Objective: To evaluate results of cervical disc arthroplasty with the Bryan prosthesis for treatment of cervicobrachial pain (radiculopathy) and spinal cord compression (myelopathy). Methods: From 2002 to 2007, the CECOL surgical staff has operated 65 patients. Only 28 patients were found in 2010 to a new data collection. The pre- and post-operative evaluation was conducted using the CSOQ questionnaire (Cervical Spine Outcomes Questionnaire). Odom criteria were used only in the postoperative evaluation. Both were translated and adapted to local culture. Results: There was a significant symptomatic and functional improvement in most patients. The reduction of neck pain (axial) and brachial pain (radicular) was similar. Odom criteria showed $82.1 \%$ good and excellent results, $10 \%$ satisfactory and $7 \%$ poor. There was only one complication (3\%) which was reversed with anterior arthrodesis. Conclusions: The total cervical disc arthroplasty has proved to be a safe and effective method to treat selected cases of cervical disc herniation with radiculopathy and/or myelopathy in the short and medium term.
\end{abstract}

Keywords: Artroplasty; Arthrodesis; Spinal cord compression; Intervertebral disk degeneration; Radiculopathy.

\section{RESUMEN}

Objetivo: Evaluar los resultados de la artroplastia discal cervical con la prótesis de Bryan para el tratamiento del radiculopatía y compresión de la médula espinal (mielopatía). Métodos: El equipo de CECOL ha operado 65 pacientes en el período de 2002 a 2007. pacientes fueron localizados en 2010 para una nueva recolección de datos. La evaluación pre y postoperatoria se realizó mediante un cuestionario CSOQ (Cervical Spine Outcomes Questionnaire). Los criterios de Odom sólo fueron utilizados en la evaluación postoperatoria. Ambos fueron traducidos y adaptados a la cultura local. Resultados: Hubo una mejora significativa sintomática y funcional en la mayoría de los pacientes. La atenuación del dolor de cuello (axial) y braquial (radicular) fue similar. Los criterios Odom demostraron 82,1\% de resultados buenos y excelentes, 10\% satisfactorios y el 7\% malos. Hubo sólo una complicación (3\%), que se revirtió con artrodesis anterior. Conclusiones: La artroplastia discal cervical ha demostrado ser un método seguro y eficaz para el tratamiento de casos seleccionados de hernia de disco cervical asociada a la radiculopatía y/o a compresión de la médula espinal en el corto y mediano plazo.

Descriptores: Artroplastia; Artrodesis; Compresión de la medula espinal; Degeneracion del disco intervertebral; Radiculopatía.

\section{INTRODUÇÃO}

A artrodese cervical anterior é a técnica considerada "padrão ouro" para tratamento cirúrgico da mielopatia, radiculopatia e dor axial cervical. No entanto há sobrecarga dos níveis adjacentes o que favorece a degeneração discal ${ }^{1-12}$. A artroplastia total disco surgiu como método alternativo de tratamento que preserva o movimento, mantém a altura discal e a estabilidade da coluna vertebral ${ }^{13,14}$.

\section{MÉTODOS}

Critérios de inclusão ${ }^{15}$ : pacientes sintomáticos com radiculopatia ou mielopatia; degeneração de um ou dois níveis entre C3 a C7; sem cirurgia cervical prévia e com mobilidade cervical. Critérios de exclusão ${ }^{15}$ : infecção ativa; osteoporose; tumores; evidências radiológicas de instabilidade mecânica ou ausência de mobilidade comprovada ao nível do tratamento nas radiografias dinâmicas pré-operatórias 
de flexão-extensão; artrose facetária cervical e os pacientes que não foram localizados para segunda fase da entrevista.

Foram operados 65 pacientes pela equipe médica da CECOL no período 28/09/2002 a 30/10/2007. No ano de 2010, somente 28 foram localizados para segunda etapa do estudo. Todos foram submetidos à artroplastia anterior com prótese Bryan após dissectomia e descompressão medular ou radicular (da coluna cervical) por via anterior

Para avaliação utilizamos o questionário CSOQ (Cervical Spine Outcomes Questionnaire $)^{16,17}$ por ser uma escala mais específica para a avaliação da dor e da função relacionadas com as doenças cervicais no pré e no pós-operatório. Os critérios Odom apud Clark ${ }^{18}$ foram utilizados somente no pós-operatorio.

A faixa etária dos 28 pacientes variou de 25 a 78 anos. Doze do sexo masculino e dezesseis do sexo feminino.

Os Critérios de Odom foram traduzidos do inglês e foram aplicados como segue:

Ótimo (excelente): melhoria na maioria (pelo menos 80\%) dos sinais e dos sintomas pré-operatórios, com pouca deterioração (não mais de 10\%). Nenhuma queixa referente à doença do disco cervical. As ocupações diárias são realizadas sem prejuízo.

Bom: melhoria em alguns (pelo menos 70\%) dos sinais e sintomas pré-operatórios, com alguma deterioração (não mais que 15\%). Desconforto intermitente relacionado à doença do disco cervical. As ocupações diárias são realizadas sem prejuízo significante.

Satisfatório: melhoria pelo menos $50 \%$ dos sinais e dos sintomas pré-operatórios, com alguma deterioração (não mais de 20\%). Melhoria subjetiva, mas as atividades físicas são significativamente limitadas. Ruim (pobre): melhoria em poucos (menos de 50\%) dos sinais e dos sintomas pré-operatórios, ou deterioração significativa (mais de $20 \%$ ). Inalterado ou pior comparado à circunstância antes da operação.

Os critérios Odom são utilizados em diversos artigos científicos pela sua especificidade para o estudo das patologias cervicais no que diz respeito à dor e a avaliação das atividades diárias. Na busca de analizar outros parâmetros como sintomas físicos, avaliação psicológica e avaliação da necessidade de fármacos além de outros questionamentos, foi escolhido o CSOQ.

O CSOQ (Cervical Spine Outcomes Questionnaire) é específico e compreensivo instrumento para avaliar a dor cervical e o resultado do tratamento, fornecendo informações sobre a severidade da dor, medição funcional, aflição psicológica, sintomas físicos e a mensuração dos cuidados a saúde. Parece aceitável para os pacientes, fácil de administrar e é altamente confiável. O CSOQ foi traduzido do inglês e utilizado como demostra o Anexo 1.

Avaliação do CSOQ: cada "score" foi transformado para contagens de 0-100. As contagens mais elevadas indicam maior disfunção corporal além de:

No I: maior severidade da experiência de dor cervical.

No II: severidade da experiência de dor ombro-braço.

No III: maior limitação funcional.

No IV: tendência a maior ansiedade, depressão.

No V: frequência mais elevada de sintomas físicos, não considerando a dor

No VI: alta frequência de utilização de mecanismos de cuidado a saúde.

\section{RESULTADOS}

Houve grande melhora na dor cervical (axial) e braquial (radicular) na maioria dos pacientes. Além disso, a recuperação funcional permitiu que a maioria deles retornasse ao trabalho, lazer, cuidados pessoais e até mesmo as atividades esportivas. A função sexual foi pouco afetada pela doença, mas obteve melhora após a cirurgia

Apenas uma complicação. A soltura do implante ocorreu após um evento traumático (queda acidental da própria altura). Nesse caso em particular foi feita conversão para artrodese anterior com ótima recuperação e sem seqüelas neurológicas, visto que a migração do implante de Bryan tende a ser anterior devido ao seu desenho, impedindo a compressão medular ou radicular ${ }^{19}$ (Figuras 1 a 10).

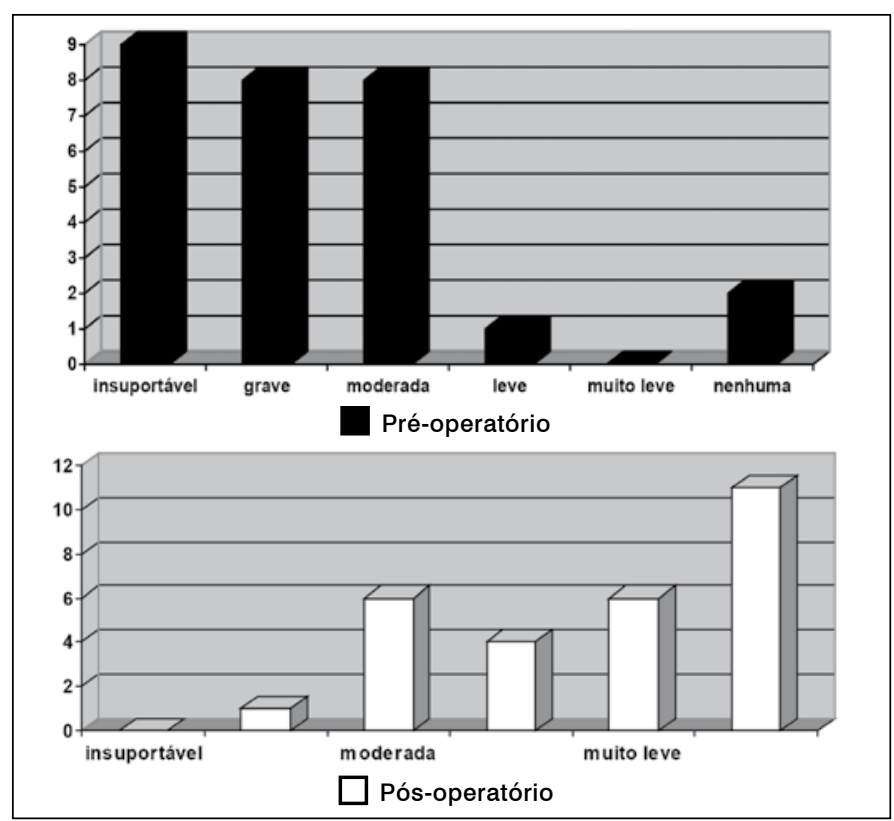

Figura 1. Severidade da dor cervical (por número absoluto de pacientes).

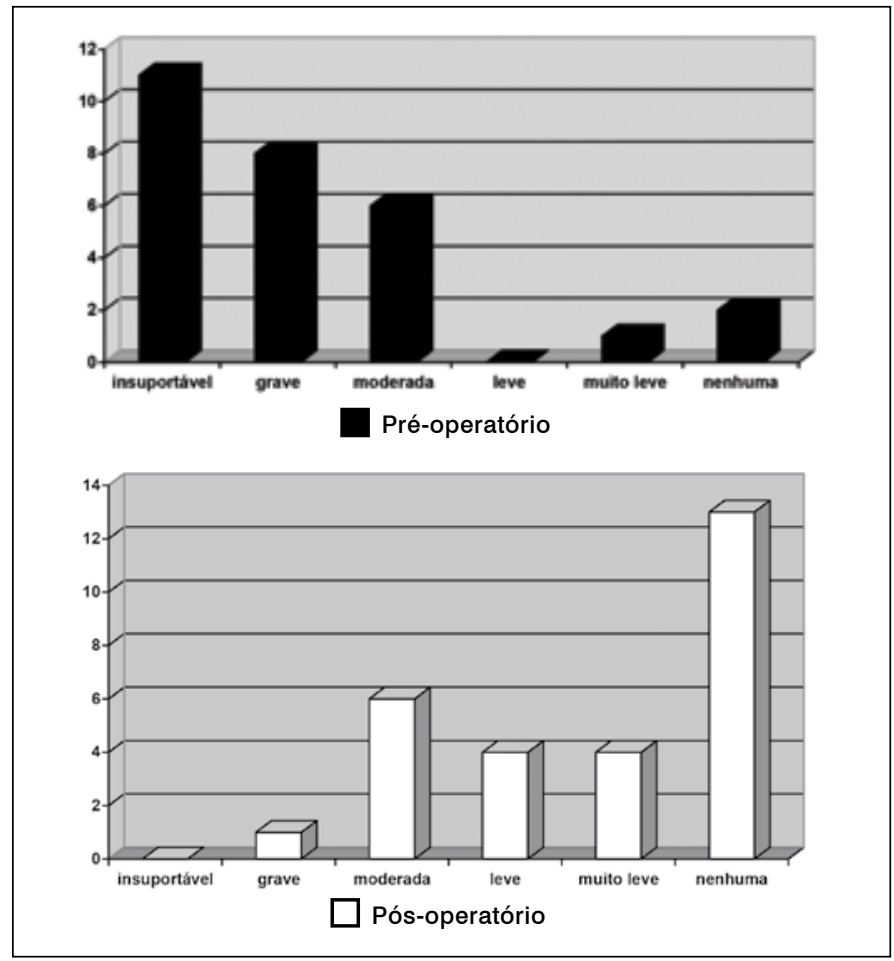

Figura 2. Severidade da dor ombro/braço (por número absoluto de pacientes)

\section{DISCUSSÃO}

Nossos resultados foram semelhantes ao de diversos estudos multicêntricos atuais. Alguns desses utilizaram a artrodese anterior após dissectomia como grupo controle e que os resultados da artroplastia em curto prazo foram iguais ou até superiores ao da artrodese ${ }^{20-24}$. Os índices de complicações, segundo eles, foram em média mais frequentes na artroplastia, no entanto foram mais graves na artrodese (lesão medular, pseudoartrose e outros) ${ }^{25}$. A ossificação heterotópica tem sido a principal causa da perda da mobilidade no nível tratado e há relatos em que até $29 \%$ dos casos foram afetados ${ }^{20}$.

Existem atualmente no mercado diversas endopróteses de substituição articular total de disco cervical confeccionadas com 


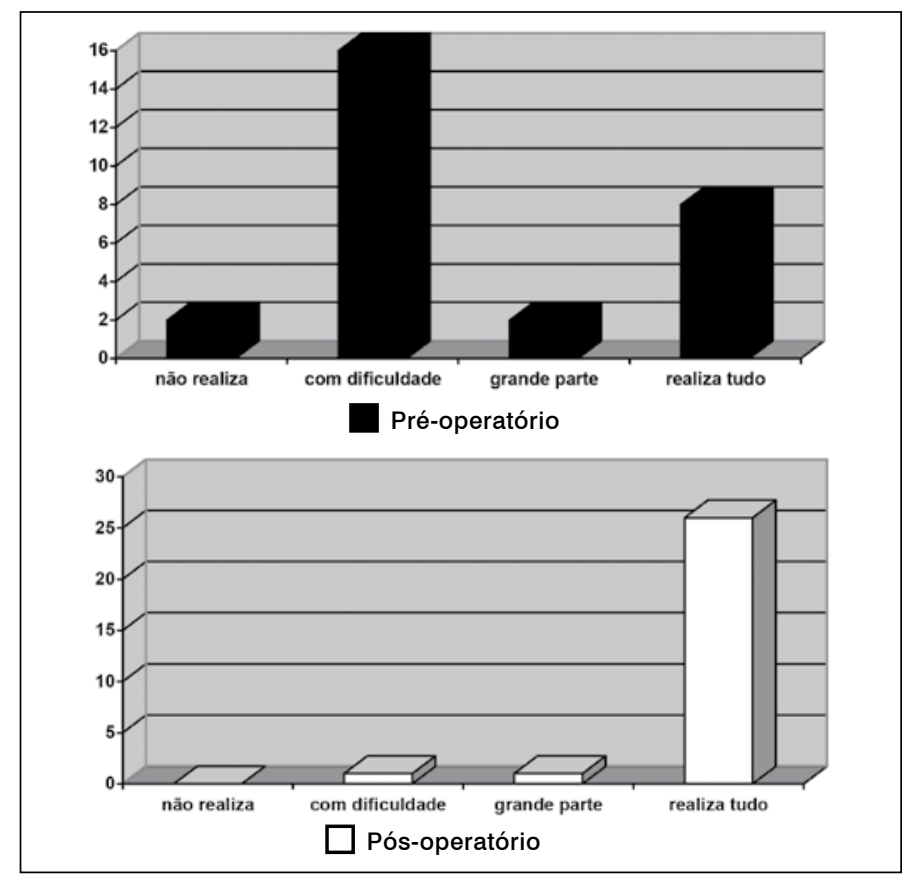

Figura 3. Medida funcional do cuidado pessoal (por número absoluto de pacientes).

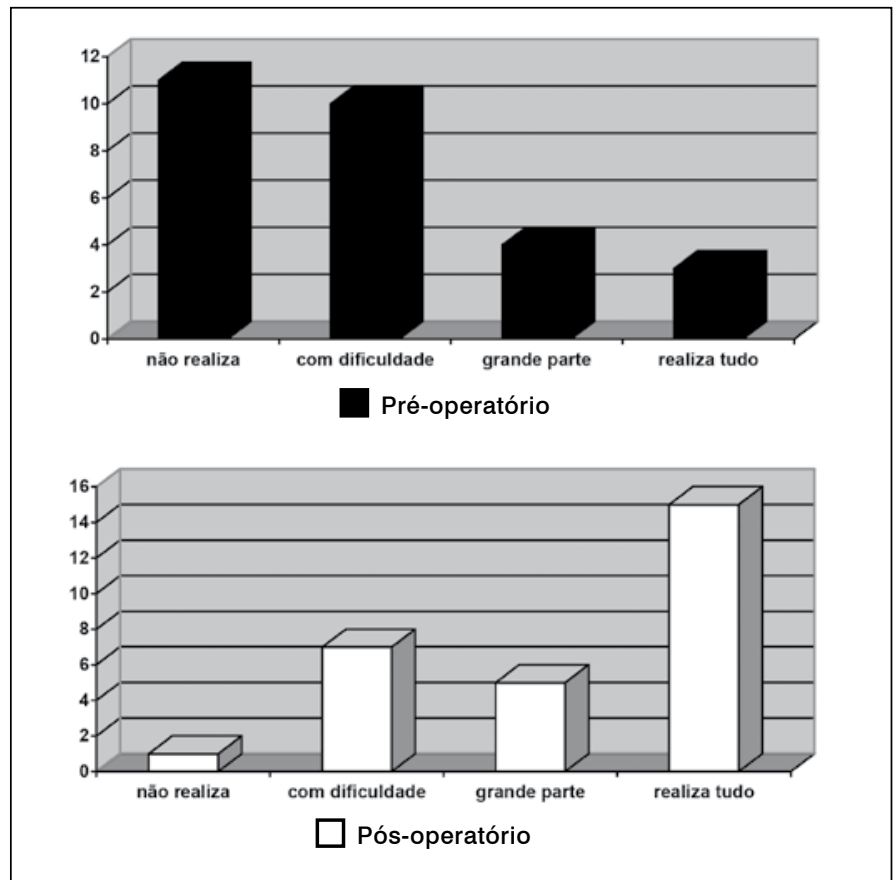

Figura 4. Medida funcional do trabalho em casa (por número absoluto de pacientes).

materiais e com desenhos diferentes (Prestige da Medtronic, Discover da DePuy, Discocerv da ScientX, Bryan da Medtronic e Prodisc C da Synthes). Tem-se observado resultados semelhantes com essas próteses em curto prazo (dois anos de seguimento) ${ }^{24,26}$.

Os fatores mais relevantes que influenciaram a recuperação segundo o Swiss Spine ${ }^{23}$ : a intensidade da dor no pré-operatório; a qualidade de vida antes da cirurgia e o uso de medicações psicotrópicas no pré-operatório. Quanto maior for à intensidade da dor no período pré-operatório maior será a melhora dos sintomas. Pacientes com a qualidade de vida muito prejudicada tendem a melhores resultados após o tratamento. O uso prévio de medicamentos psicotrópicos foi associado a resultados pobres.

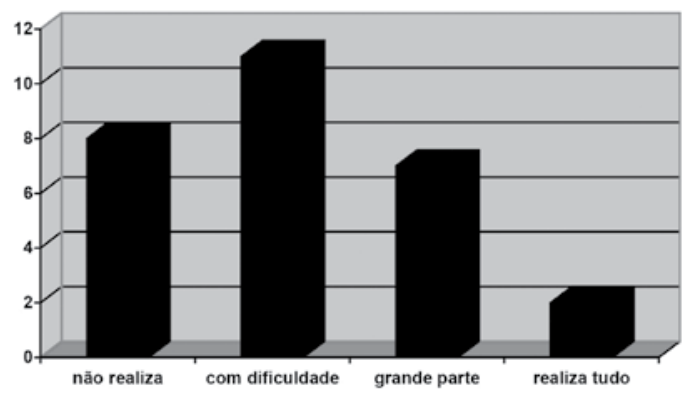

Pré-operatório

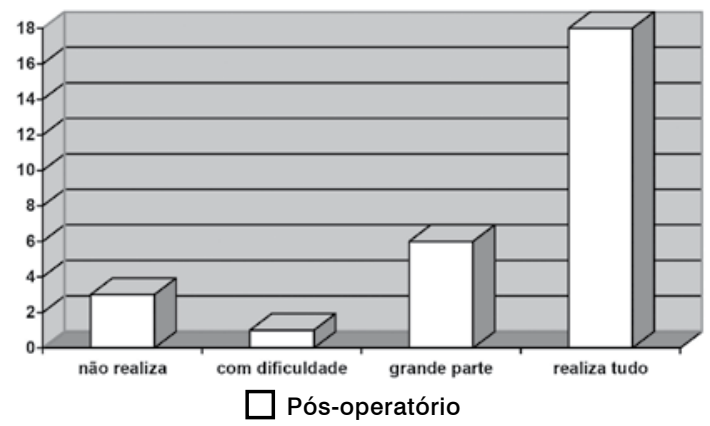

Figura 5. Medida funcional: recreação (por número absoluto de pacientes).

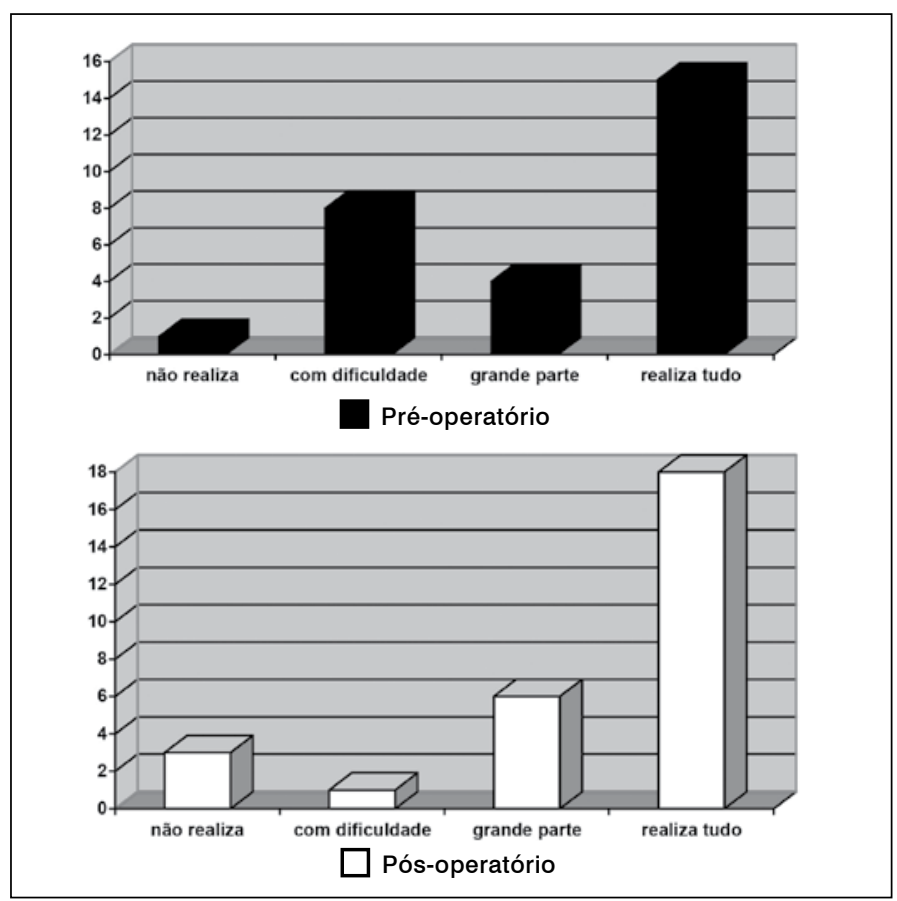

Figura 6. Medida funcional: atividade sexual (por número absoluto de pacientes).

No tratamento da mielopatia havia um conceito teórico antigo de que a preservação do movimento manteria o microtrauma de repetição ${ }^{27}$ na medula espinhal lesionada afetando de forma negativa os resultados. Na prática a artroplastia foi tão eficaz quanto a artrodese ${ }^{26}$. Na nossa casuística tivemos um único caso de mielopatia e a recuperação foi satisfatória com a artroplastia.

Muitos pacientes operados são relativamente jovens e ainda não sabemos qual será a evolução após 20, 30 anos ou mais. Através da experiência acumulada das artroplastias de joelho e quadril imaginamos a possibilidade de falência dos implantes por: quebra devido a fadiga, soltura da interface osso/implante e afundamento na placa terminal, todas com possibilidade de correção com artro- 


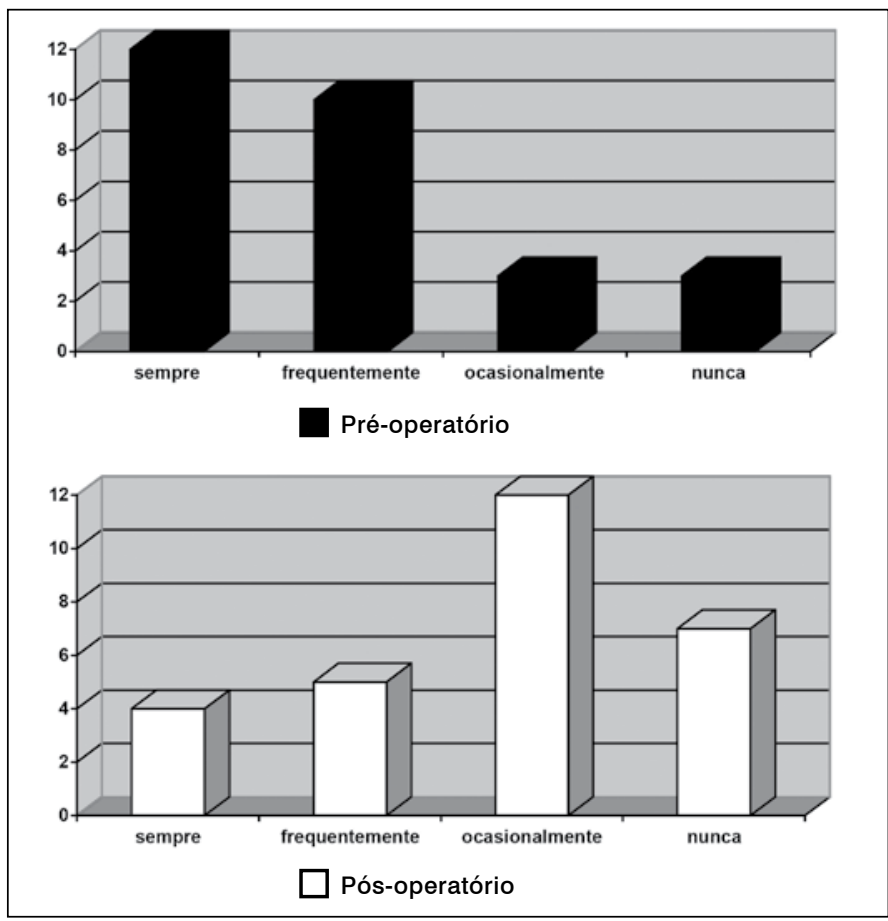

Figura 7. Medida psicológica da aflição (por número absoluto de pacientes).

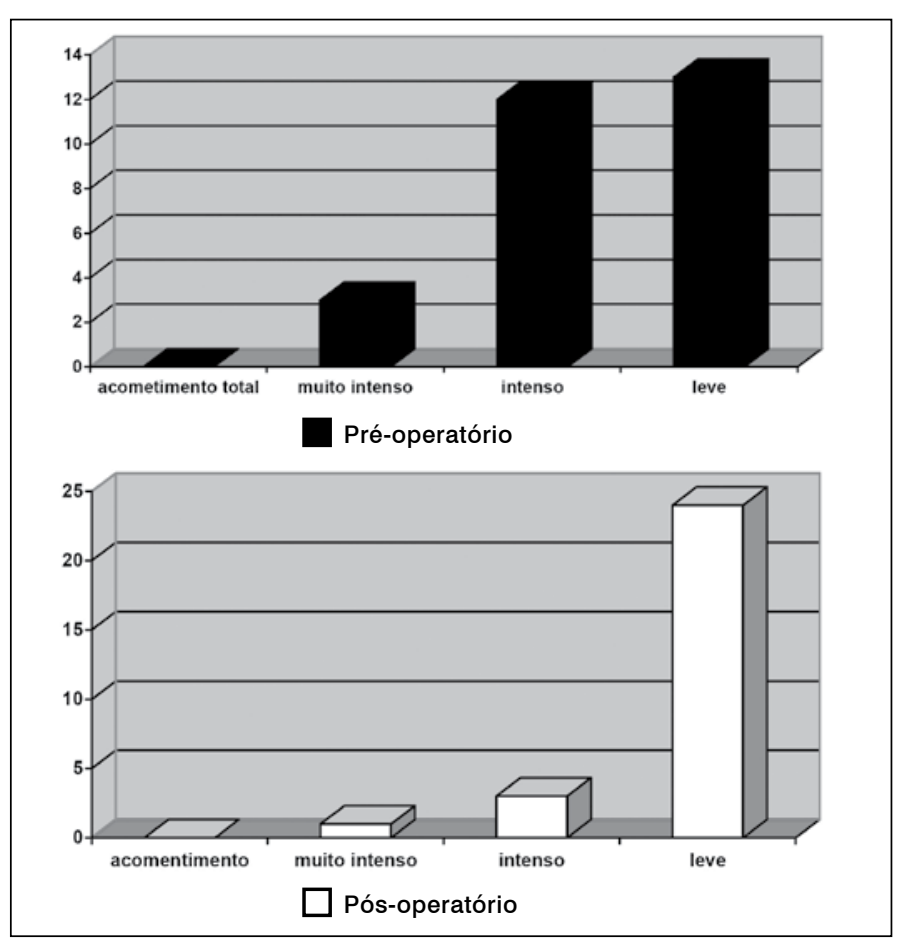

Figura 8. Medidas físicas dos sintomas (por número absoluto de pacientes).

dese anterior, tendo poupado a mobilidade cervical durante anos ou décadas. Outra possibilidade (já observada) seria a da ossificação heterotópica e anquilose com provável resolução espontânea. Essas hipóteses só serão confirmadas e a durabilidade média dessas endopróteses só serão definidas após seguimentos de longo prazo.

\section{REFERÊNCIAS}

1. Cherubino P, Benazzo F, Borromeo U, Perle S. Degenerative arthritis of the adjacent spinal joints following anterior cervical spinal fusion: clinicoradiologic and statistical correlations. Ital J Orthop Traumatol. 1990;16(4):533-43.

2. Clements DH, O'Leary PF. Anterior cervical discectomy and fusion. Spine (Phila Pa 1976).

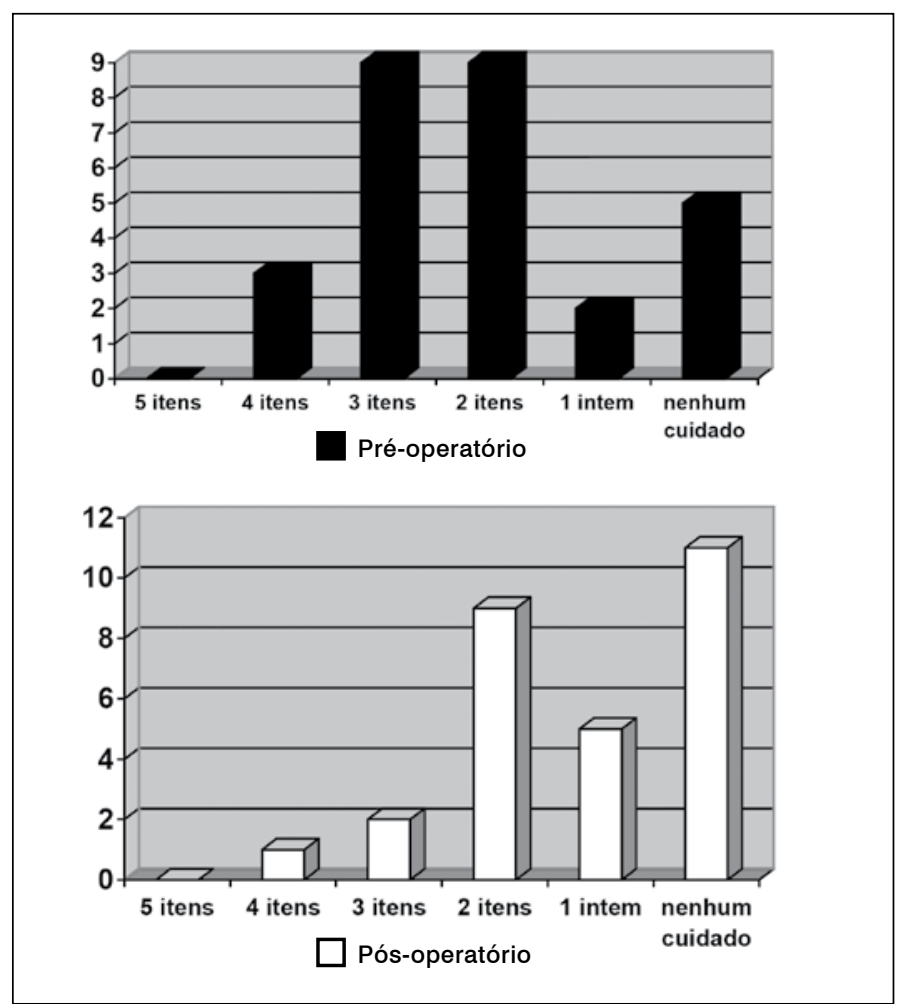

Figura 9. Mensurações dos cuidados a saúde (por número absoluto de pacientes).

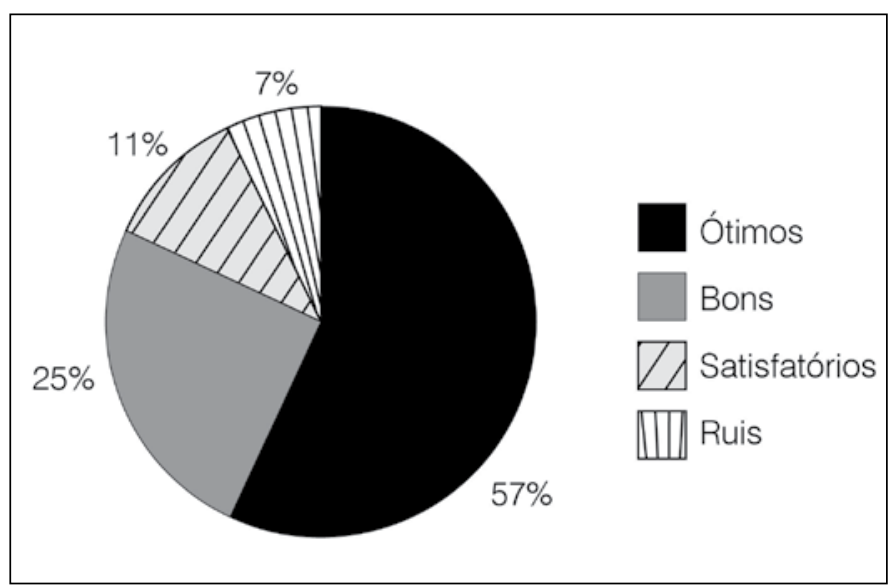

Figura 10. Critérios de ODOM (por percentual).

\section{CONCLUSÃO}

A artroplatia total de disco cervical demonstrou se um método de tratamento seguro e eficaz para casos selecionados de discopatia degenerativa e hérnia discal cervical e mielopatia a curto e médio prazo.

\section{AGRADECIMENTOS}

Agradecemos à Sra.Claudia Albuquerque, administradora da CECOL e o Sr. Fábio Faria, administrador da CECOL pelo apoio na realização deste trabalho.

3. Döhler JR, Kahn MR, Hughes SP. Instability of the cervical spine after anterior interbody fusion. A study on its incidence and clinical significance in 21 patients. Arch Orthop Trauma Surg. 1985;104(4):247-50. 
4. Goffin J, van Loon J, Van Calenbergh F, Plets C. Long-term results after anterior cervical fusion and osteosynthetic stabilization for fractures and/or dislocations of the cervical spine. J Spinal Disord. 1995:8(6):500-8.

5. Hilibrand AS, Carlson GD, Palumbo MA, Jones PK, Bohlman HH. Radiculopathy and myelopathy at segments adjacent to the site of a previous anterior cervical arthrodesis. J Bone Joint Surg Am. 1999:81(4):519-28.

6. Matsunaga S, Kabayama S, Yamamoto T, Yone K, Sakou T, Nakanishi K. Strain on intervertebral discs after anterior cervical decompression and fusion. Spine (Phila Pa 1976). 1999:24(7):670-5.

7. Pospiech J, Stolke D, Wilke HJ, Claes LE. Intradiscal pressure recordings in the cervical spine. Neurosurgery. 1999;44(2):379-84.

8. Woesner ME, Mitts MG. The evaluation of cervical spine motion below C2: a comparison of cineroentgenographic and conventional roentgenographic methods. Am J Roentgenol Radium Ther Nucl Med. 1972;115(1):148-54

9. Goffin J, Van Calenbergh F, van Loon J, Casey A, Kehr P, Liebig K, et al. Intermediate follow-up after treatment of degenerative disc disease with the Bryan Cervical Disc Prosthesis: single-level and bi-level. Spine (Phila Pa 1976). 2003;28(24):2673-8.

10. Sears W. Bryan cervical disc arthroplasty: 12 month minimum follow-up-in 14 patients. $J$ Bone Joint Surg Br. 2004:86(Suppl 4):461.

11. Theisen IJ, Theisen DM, Oliveira MAA, Berwanger CGW. Comparação de quatro técnicas cirurgicas para discectomia cervical anterior e artrodese. Coluna/Columna. 2004:3(3):117-21.

12. Anderson PA, Rouleau JP, Bryan VE, Carlson CS. Wear analysis of the Bryan Cervical Disc prosthesis. Spine (Phila Pa 1976). 2003;28(20):S186-94.

13. Goffin J, Casey A, Kehr P, Liebig K, Lind B, Logroscino C, et al. Preliminary clinical experience with the Bryan Cervical Disc Prosthesis. Neurosurgery. 2002;51(3):840-5.

14. Wang MY, Leung $\mathrm{CH}$, Casey AT. Cervical arthroplasty with the Bryan disc. Neurosurgery 2005;56(Suppl 1):58-65

15. Herkowitz HN. Garfin SR, Eismont FJ, Bell GR, Balderston RA, editors. Rothman-Simeone the spine. 5th ed. Philadelphia: Saunders/Elsevier; 2006.
16. BenDebba M, Heller J, Ducker TB, Eisinger JM. Cervical spine outcomes questionnaire: its development and psychometric properties. Spine (Phila Pa 1976). 2002;27(19):2116-23.

17. Lafuent JB. El implante discal cervical como alternative a la artrodesis em el tratamiento quirúrgico de la cervicoartrosis [tesis]. Barcelona: Universitat Autônoma de Barcelona; 1994

18. Clark C, editor. The cervical spine research society. 4th ed. Phildelphia: Lippincott Wiliams \&Wilkins; 2004

19. Pickett GE, Sekhon LH, Sears WR, Duggal N. Complications with cervical arthroplasty. J Neurosurg Spine. 2006;4(2):98-105.

20. Sasso RC, Smucker JD, Hacker RJ, Heller JG. Artificial disc versus fusion: a prospective, randomized study with 2-year follow-up on 99 patients. Spine (Phila Pa 1976). 2007;32(26):2933-40.

21. Heidecke V, Burkert W, Brucke M, Rainov NG. Intervertebral disc replacement for cervica degenerative disease-clinical results and functional outcome at two years in patients implanted with the Bryan cervical disc prosthesis. Acta Neurochir (Wien). 2008;150(5):453-9.

22. Sun Y, Zhao YB, Zhou FF, Liu ZJ. [The application of cervical spine arthroplasty with Bryan disc and mid-term clinical and radiological follow-up results]. Zhonghua Wai Ke Za Zhi. 2008;46(5):333-7.

23. Zechmeister I, Winkler R, Mad P. Artificial total disc replacement versus fusion for the cervical spine: a systematic review. Eur Spine J. 2011;20(2):177-84.

24. Schluessmann E, Aghayev E, Staub L, Moulin P, Zweig T, Röder C. SWISSspine: the case of a governmentally required HTA-registry for total disc arthroplasty: results of cervical disc prostheses. Spine (Phila Pa 1976). 2010;35(24):E1397-405.

25. Anderson PA, Sasso RC, Riew KD. Comparison of adverse events between the Bryan artificial cervical disc and anterior cervical arthrodesis. Spine (Phila Pa 1976) 2008;33(12):1305-12

26. Lazaro BC, Yucesoy K, Yuksel KZ, Kowalczyk I, Rabin D, Fink M, et al. Effect of arthroplasty design on cervical spine kinematics: analysis of the Bryan Disc, ProDisc-C, and Synergy disc. Neurosurg Focus. 2010;28(6):E6.

27. Zindrick M, Harris MB, Humphreys SC, O'Leary PT, Schneiderman G, Watters WC 3rd, et al. Cervical disc arthroplasty. J Am Acad Orthop Surg. 2010;18(10):631-7.

Anexo 1. CSOQ em português.

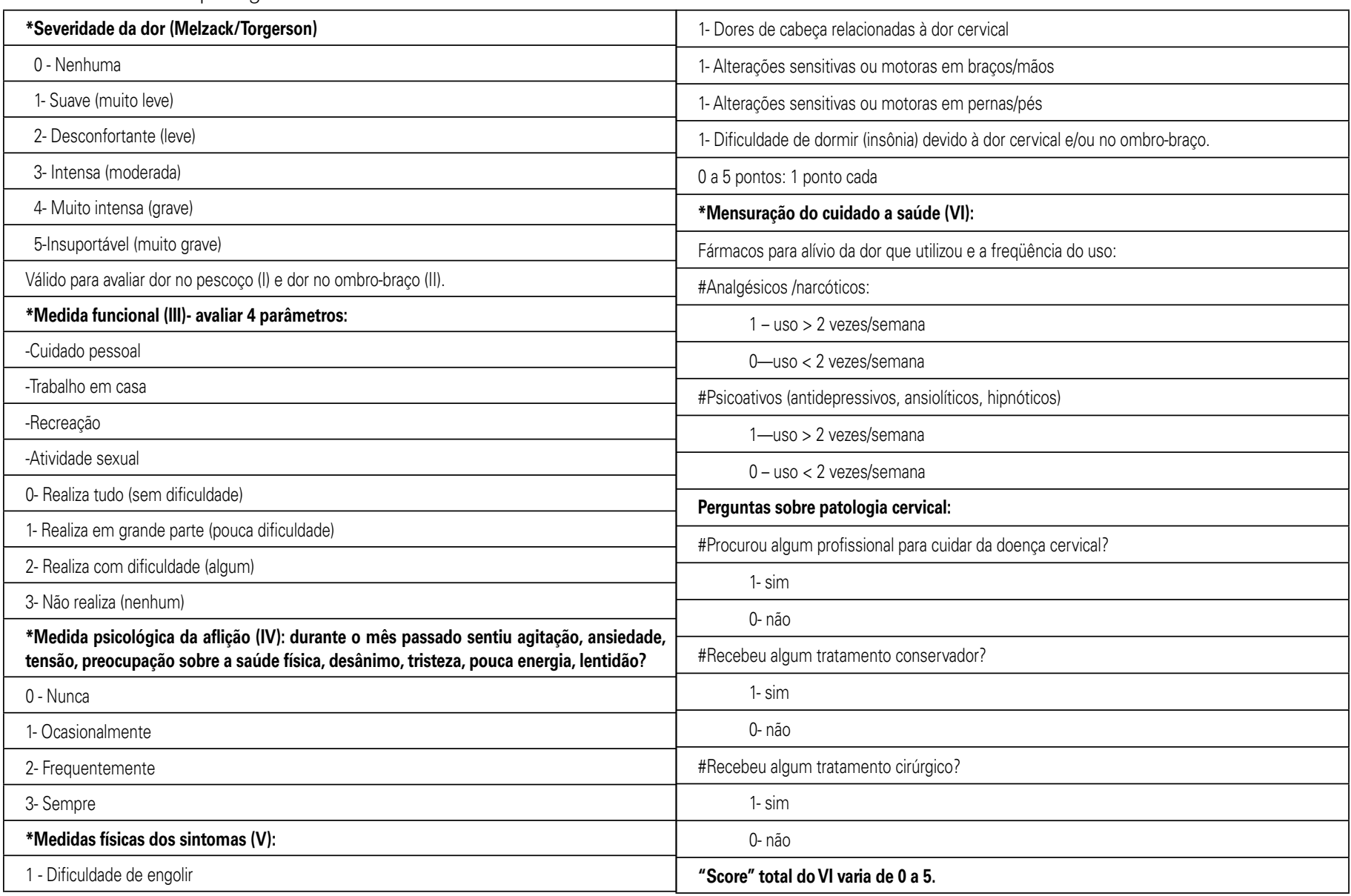

